\title{
Paratesticular adenocarcinoma: unusual presentation of metastasis of pancreatic cancer
}

\author{
Janja Ocvirk and Boštjan Šeruga \\ Department of Medical Oncology, Institute of Oncology, Ljubljana, Slovenia
}

\begin{abstract}
Background. Metastatic paratesticular adenocarcinoma from the pancreatic cancer is very rare. To our knowledge, there are less than 20 cases published in the literature.

Case report. We experienced a case of paratesticular adenocarcinoma from the primary pancreatic cancer. A 42-year-old man was presented with locoregionally advanced carcinoma of the tail of the pancreas with intraoperatively found liver metastases and with a tumour in the right hemi-scrotum. Ultrasound of the scrotum revealed a paratesticular tumour. A fine needle aspiration biopsy (FNAB) confirmed a poorly differentiated adenocarcinoma and it was in concordance with the diagnosis of the primary tumour. The patient started treatment with chemotherapy with gemcitabine. Unfortunately, he progressed one month later and the treatment was discontinued.
\end{abstract}

Conclusions. Outcome in the adenocarcinoma of the pancreas is dismal. The only possible treatment option for metastatic disease is systemic therapy but the results are disappointing, as in the present case.

Key words: pancreatic neoplasms; neoplasms metastasis; testicular neoplasms - secondary

\section{Introduction}

Pancreatic cancer can be silent for a long time before it manifests with features related to local and distant spread. Paratesticular metastases of pancreatic cancer are unusual tumours. Generally, paratesticular tumours are rare. Primary malignant tumours and metastatic tumours account for $32.9 \%$ of all tumours and $6-8 \%$ of malignant tumours of paratesticular tissue, respectively. In $47.6 \%$ of the cases,

Received 19 March 2007

Accepted 26 March 2007

Correspondence: Boštjan Šeruga, M.D., Institute of Oncology Ljubljana, Dept. of Medical Oncology, Zaloska c. 2, 1000 Ljubljana, Slovenia; Phone +386 15879 103; Fax: +386 15879 400: E-mail: bseruga@onko-i.si the metastases and the primary tumours are found simultaneously. Uncommonly, in 9.5\% they are the first sign of occult cancer. The most common primary sites of metastasis of the paratesticular tissue are prostate, kidney, gastrointestinal tract, lung and breast cancer. ${ }^{1,2}$. We report a case of simultaneous metastatic paratesticular adenocarcinoma originating in the tail of the pancreas.

\section{Case presentation and management}

A 42-year-old man was presented to abdominal surgeon with recurrent vague upper $a b-$ dominal discomfort lasting for few months and without significant weight loss. His past history was not remarkable. Abdominal and endoscopic ultrasound examinations 
revealed a suspicious cystic and nonhomogeneous lesion in the tail of the pancreas. Chest X-ray was negative for metastasis. The abdominal CT scan confirmed a $2 \mathrm{~cm}$ cystic lesion in the tail of the pancreas. The serum tumour marker antigens $\mathrm{Ca}$ 19-9 and CEA were elevated, $78 \mathrm{U} / \mathrm{ml}$ and $2.6 \mathrm{ng} / \mathrm{ml}$, respectively. Explorative laparatomy was done and locoregionally inoperable tumour of the pancreas with multiple liver metasteases was found. Histologically, moderately differentiated adenocarcinoma from the pancreas and the liver was confirmed.

Afterwards, the patient was presented to the medical oncologist and he complained of palpable tumour mass in the right hemi-scrotum. On examination a painless, hard and irregular $2 \times 2 \mathrm{~cm}$ large swelling was identified. Ultrasound revealed a paratesticular tumour. A fine needle aspiration biopsy (FNAB) confirmed a poorly differentiated adenocarcinoma.

Tumour marker antigens Ca 19-9 and CEA were further elevated, $296 \mathrm{U} / \mathrm{ml}$ and $15.4 \mathrm{ng} / \mathrm{ml}$, respectively. Based on these findings, the patient was diagnosed as having a metastatic paratesticular adenocarcinoma originating from pancreatic carcinoma with liver metasteses.

He started treatment with chemotherapy with gemcitabine. Unfortunately, he progressed one month later and the treatment was discontinued.

\section{Discussion}

Metastatic paratesticular adenocarcinoma from the pancreatic cancer is very rare. To our knowledge, there are less than 20 cases published in the literature. In most cases the primary tumour was located in the body or the tail of the pancreas what is in concordance with the present case. ${ }^{3-6}$

Results from the 154 consecutive autopsies of the patients with pancreatic adenocarcinoma revealed that carcinomas of the body and/or tail of the pancreas were more frequent characterized by transperitoneal and hematogenous dissemination than carcinomas of the head of the pancreas. ${ }^{7}$ Kamisawa et al have sugested a mechanisem of unusual pattern of spread due to hepatofugal portosystemic shunting induced by splenic vein obstruction, retrograde lymphatic infiltration or even aggressive tumour characteristics. ${ }^{8}$ In the present case, we postulate a hematogenous route of dissemination to the paratesticular tissue because of the presence of liver metastases without any metastatic lymph nodes.

Outcome in the adenocarcinoma of the pancreas is dismal with a five-year survival rate of $4 \% .{ }^{9}$ The only possible treatment option for metastatic disease is systemic therapy but the results are disappointing, as in the present case.

\section{References}

1. Algaba F, Santaularia JM, Villavicencio $H$. Metastatic tumor of the epididymis and spermatic cord. Eur Urol 1983; 9: 56-9.

2. Beccia DJ, Krane RJ, Olsson CA. Clinical management of non-testicular intrascrotal tumors. J Urol 1976; 116: 476-9.

3. Tanaka H, Yasui T, Watase H. Metastatic tumor of the epididymis from pancreatic carcinoma: a case report. [Japanese]. Acta Urol Jpn 1999; 45: 649-52.

4. Sawa TE, Duun S, Andersen JT. Paratesticular tumor: a metastasis from primary pancreas cancer. Scand J Urol Nephrol 2000; 34: 70-1.

5. Seo IY, Kim SG, Han WC, Rim JS. Paratesticular mucinous cystadenocarcinoma: metastasis from pancreatic cancer. Int J Urol 2004; 11: 1147-9.

6. Dookeran KA, Lotze MT, Sikora SS, Rao UN. Pancreatic and ampullary carcinomas with intrascrotal metastases. Br J Surg 1997; 84: 198-9.

7. Mao C, Domenico DR, Kim K, Hanson DJ, Howard JM. Observations on the developmental patterns and the consequences of pancreatic exocrine adenocarcinoma. Findings of 154 autopsies. Arch Surg 1995; 130: 125-34.

8. Kamisawa T, Isawa T, Koike M, Tsuruta K, Okamoto A. Hematogenous metastases of pancreatic ductal carcinoma. Pancreas 1995; 11: 345-9.

9. Brenner $H$. Long-term survival rates of cancer patients achieved by the end of the $20^{\text {th }}$ century: a period analysis. Lancet 2002; 360: 1131-5. 